\title{
Neurological manifestations of COVID-19, SARS and MERS
}

\author{
Kato Verstrepen $^{1,2} \cdot$ Laure Baisier $^{1,2} \cdot$ Harald De Cauwer $^{1,2} \mathbb{C}$
}

Received: 5 April 2020 / Accepted: 12 June 2020 / Published online: 21 July 2020

(c) Belgian Neurological Society 2020

\begin{abstract}
Since December 2019, the world is affected by an outbreak of a new disease named COVID-19, which is an acronym of 'coronavirus disease 2019'. Coronaviruses (CoV) were assumed to be associated with mild upper respiratory tract infections, such as common cold. This perception changed in time due to occurrence of the Severe Acute Respiratory Syndrome (SARS) caused by SARS-CoV in 2002 and the Middle East Respiratory Syndrome (MERS) caused by MERS-CoV in 2012, both inducing an epidemic severe viral pneumonia with potentially respiratory failure and numerous extra-pulmonary manifestations. The novel coronavirus, SARS-CoV-2, is likewise a causative pathogen for severe viral pneumonia with the risk of progression to respiratory failure and systemic manifestations. In this review, we will give a summary of the neurological manifestations due to SARS and MERS, as those might predict the neurological outcome in the novel COVID-19. Additionally, we provide an overview of the current knowledge concerning neurological manifestations associated with COVID-19, to the extent that literature is already available as the pandemic is still ongoing.
\end{abstract}

Keywords Neurology $\cdot$ COVID-19 $\cdot$ SARS $\cdot$ MERS $\cdot$ Stroke $\cdot$ Neuropathy

\section{Introduction}

Viruses of the Coronaviridae family are positive-sensed, single-stranded RNA viruses. They are broadly distributed in different animal species including avian host, cats, dogs, bats, camels, cattle and mice. Among these viruses, some are pathogenic to human [1-3].

In humans, $\mathrm{CoV}$ infections were primarily associated with upper respiratory tract and gastrointestinal tract infections. However, the last 2 decades the world was affected by several viral epidemics, such as Severe Acute Respiratory Syndrome Coronavirus (SARS-CoV) in 2002-2003 and Middle East Respiratory Syndrome Coronavirus (MERS$\mathrm{CoV}$ ) in 2012 , both resulting in high mortality rate, respectively, $10 \%$ and $35 \%$.

Since December 2019, the world is affected by an outbreak of a new disease named COVID-19, which is an acronym of 'coronavirus disease 2019'. It is caused by a novel

Harald De Cauwer

harald.decauwer@ziekenhuisgeel.be

1 Department of Neurology, Dimpna Regional Hospital, JB Stessenstraat 2, 2440 Geel, Belgium

2 Faculty of Medicine and Health Sciences, University of Antwerp, Wilrijk, Belgium coronavirus (CoV), named SARS-CoV-2, due to similarities with the Severe Acute Respiratory Syndrome Coronavirus (SARS-CoV) [1].

All three infections show a broad spectrum of clinical manifestation, varying from asymptomatic or mild disease to severe illness with risk of progress to respiratory failure due to viral pulmonary infection $[4,5]$.

It is known that human coronaviruses can reach the central nervous system (CNS) and that they could be associated with neurological symptoms [6]. Several cases of neurological involvement during SARS and MERS and the potential mechanisms have already been described in literature [4-7].

Conversely, despite the current global outbreak with many more patients affected, little is known about neurological manifestations in COVID-19 after 6 months. In this review, we will give an overview of these neurological manifestations reported due to SARS and MERS as this might be of great importance in dealing with the novel COVID-19. Additionally, we present a summary of the current knowledge-still evolving in literature-on neurological manifestations associated with SARS-CoV-2-infection. 


\section{Method}

\section{Study selection}

The authors searched PubMed/MEDLINE databases in March 2020. Articles related to the topic were identified by following terms: "Severe Acute Respiratory Syndrome", "Middle East Respiratory Syndrome”, Coronavirus disease 2019”, "Neurology", "MERS”, "SARS”, “COVID-19”, "Stroke”, "Epilepsy", "Guillain-Barré Syndrome”, "Encephalitis", "Myelitis", "Meningitis", "Neurological Sequels", "Polyneuropathy" and "Carotid Dissection".

We used a date restriction ranging from the $1^{\text {st }}$ of January 2002 until present. There were limited linguistic restrictions (articles in English, Dutch, French and German were eligible for inclusion).

"Middle East Respiratory Syndrome" and "Neurology" identified 53 articles, of which 20 articles were retained based on review of title and abstract to select material for potential review. "Severe Acute Respiratory Syndrome" and "Neurology" revealed 102 articles, "Coronavirus disease 2019 and Neurology" revealed 1 article, "MERS" and "Neurology" 109 articles, "SARS" and "Neurology" 25 articles, "COVID19" and "Neurology" 5 articles, ("SARS" OR "MERS" OR "COVID-19") and "Stroke" 17 articles, ("SARS" OR "MERS" OR "COVID-19”) and "Epilepsy" 15 articles, ("SARS" OR "MERS" OR “COVID-19") and Guillain-Barré syndrome" 3 articles, ("SARS" OR "MERS" OR “COVID-19”) and "Myelitis" 23 articles, ("SARS" OR "MERS" OR "COVID19") and "Carotid dissection" 1 articles, but after reviewing the titles and abstracts, no additional articles were retained. ("SARS" OR "MERS" OR “COVID-19") and "Encephalitis" revealed 252 articles, of which 6 articles were selected for the review based on title and abstract. ("SARS" OR "MERS" OR "COVID-19") and "Meningitis revealed 45 articles, of which 1 article was a potential result for the review. However, this article was only accessible in Danish and was not retained for this review. ("SARS" OR "MERS" OR “COVID-19”) and "Neurological sequels" revealed 47 articles, of which 3 were selected for the review. ("SARS" OR "MERS" OR "COVID19") and "Polyneuropathy" delivered 7 results, of which 1 was retained.

The manuscripts that were considered as suitable for the review were evaluated via full text review. Interesting articles for our review noticed in the references of these articles, were used for additional information.

\section{Results}

\section{Are coronaviruses related with neuro-inflammatory disease?}

Human coronaviruses ( $\mathrm{HCoV}$ ) are known to have neurotropic and neuro-invasive capabilities. Desforges et al. hypothesize human coronaviruses are neurovirulent, as they could contribute in short- and long-term neurological disorders such as encephalomyelitis and multiple sclerosis [6-8]. The presence of HCoV RNA in the human CNS confirms these properties [9].

Viruses, in general, may enter the brain and spinal cord via hematogenous or retrograde neuronal distribution. It is already known that $\mathrm{HCoV}$ can also spread from the respiratory tract to the central nervous system (CNS) through transneuronal and hematogenous routes [10].

$\mathrm{HCoV}$ may persist in human brains, so it is assumed that long-term sequelae can develop related to emergence or aggravation of chronic neurological diseases, as it is already described for the human coronaviruses HCOV229E and HCOV-OC43. The latter have been detected in various neurological diseases, including multiple sclerosis [9]. Several recent articles report associated cases of encephalitis, acute flaccid paralysis and other neurological symptoms, such as Guillain-Barré syndrome or ADEM, as possible complications of a $\mathrm{HCoV}$ infection [6].

In this article, we will focus on the neurological manifestations described in SARS, MERS, and COVID-19.

\section{Initial clinical signs of SARS, MERS, and COVID-19 include non-specific neurological symptoms}

SARS may present with non-specific symptoms such as persistent fever, non-productive cough, myalgia, dyspnoea and headache. In roughly $20 \%$ of the patients, the initial presentation got complicated with acute respiratory distress syndrome [6]. Aside from the respiratory tract, SARS-CoV infection may also target intestinal mucosal cells, renal tubular epithelial cells and neurons as well as cells from the lymphoid and reticuloendothelial system. As a consequence, extrapulmonary features including watery diarrhoea and vomiting may also be part of the clinical presentation. Furthermore, dizziness, nausea, decrease in general well-being and confusion have also been reported [5].

The clinical presentation of MERS may also be nonspecific. Runny nose, sore throat, low-grade fever and myalgia can present before the viremia becomes detectable [4, 11]. In case of severe disease, progression to acute respiratory distress may occur. Extrapulmonary 
manifestations including gastrointestinal symptoms and acute kidney failure have been reported in case of severe illness, as well as neurological manifestations [11].

Common clinical features of COVID-19 include fever, cough, sore throat, headache, fatigue, myalgia, anosmia and dyspnoea. The infection may evolve into pneumonia, respiratory failure, multiple organ failure and death due to extreme rise in inflammatory cytokines $[1,12]$.

\section{Neurological manifestations during evolving SARS}

Current data, mainly from Chinese brain autopsy studies, confirm CNS invasion by SARS-CoV [13, 14].

Cases described in the articles mentioned below concerning neurological manifestations reported during SARS-CoVinfection are rendered in Table 1 [3].

\section{Epilepsy}

Hung et al. described the first evidence of entry of SARS$\mathrm{CoV}$ in cerebrospinal fluid (CSF) causing seizures. They reported a case of a 59-year-old woman, who experienced a status epilepticus associated with SARS. Both serum and CSF tested positive for SARS-RNA by RT-PCR [15].

Lau et al. reported a case of a 32-year-old female who developed a generalized tonic-clonic insult on day 22 of illness with SARS-CoV being detected in the CSF by RTPCR [7].

Another impact of the SARS-epidemic was the adverse effect on seizure control due to withdrawal of anti-epileptic drug treatment as many patients avoided hospital visits [16].

\section{Neuromuscular disorders}

Neuromuscular disorders in SARS are predominantly lateonset sequelae and include critical-illness polyneuropathy and myopathy [17].

Muscle weakness and elevated serum creatine kinase levels occur in more than $30 \%$ of patients with SARS [18]. In patients with fatal SARS, Leung et al. examined post-mortem histological muscle samples and described a myopathy, either resulting from critical illness myopathy or from the immune response against SARS-CoV [19].

Critical illness myopathy and critical illness polyneuropathy can both occur in a context entitled the systemic inflammatory response syndrome (SIRS). SIRS is a term comprising sepsis and multiple organ failure, which can both be aspects of SARS-CoV-infections and be related to excessive production of tumour necrosis factor alfa and nitric oxide in the macrophages [20-22]

Rhabdomyolysis has previously been associated with many infectious diseases, but it is rarely seen in SARS-CoVinfection [23]. In a case series, rhabdomyolysis correlated with renal failure and was considered to be a severe manifestation of SARS. Therefore, creatine kinase levels of SARSpatients should be monitored thoroughly [24].

\section{Olfactory neuropathy}

In contrast to frequent occurrence of anosmia in early stages of COVID-19 (see below), it is anecdotal in SARS and only described in late stage of the disease. Nevertheless, olfactory function test should be part of routine work-up for patients with SARS [25].

\section{Stroke}

Five cases of large artery ischaemic stroke were described in SARS. It is suggested that a pro-coagulant state could be present in patients with SARS [26, 27]. This could contribute to large cerebral arterial thromboembolism, together with factors as systemic hypotension and cardiac dysfunction. However, stroke is not uncommon in critically ill patients with co-morbidity. Furthermore, it is believed that the use of intravenous immunoglobulins, used to treat SARS, could play a role in SARS-related stroke. Hyperviscosity induced by intravenous immunoglobulins in patients already prone to hypercoagulable state due to viral infection and the inflammatory response, could play a key role in stroke [26, 27].

\section{Chronic post-SARS syndrome and autonomic dysfunction}

Chronic post-SARS syndrome was described by Moldofsky et al. The syndrome is characterized by persistent fatigue, diffuse myalgia, weakness, depression, nonrestorative sleep with associated REM-related apneas/hypopneas, an elevated sleep EEG cyclical alternating pattern and alpha EEG sleep anomaly [28].

Other authors suggest that prolonged fatigue and malaise may be linked to peripheral and autonomic nervous system dysfunction. Chronic fatigue may be present months after recovery from acute illness. One study shows autonomic dysfunction to be present in $50 \%$ of recovered SARSpatients. Subclinical orthostatic hemodynamic disturbances would lead to fatigue and dizziness $[29,30]$.

\section{Neurological manifestations associated with MERS-CoV infection}

Cases described in the articles mentioned below concerning neurological manifestations reported during MERS-CoVinfection are rendered in Table 2. 


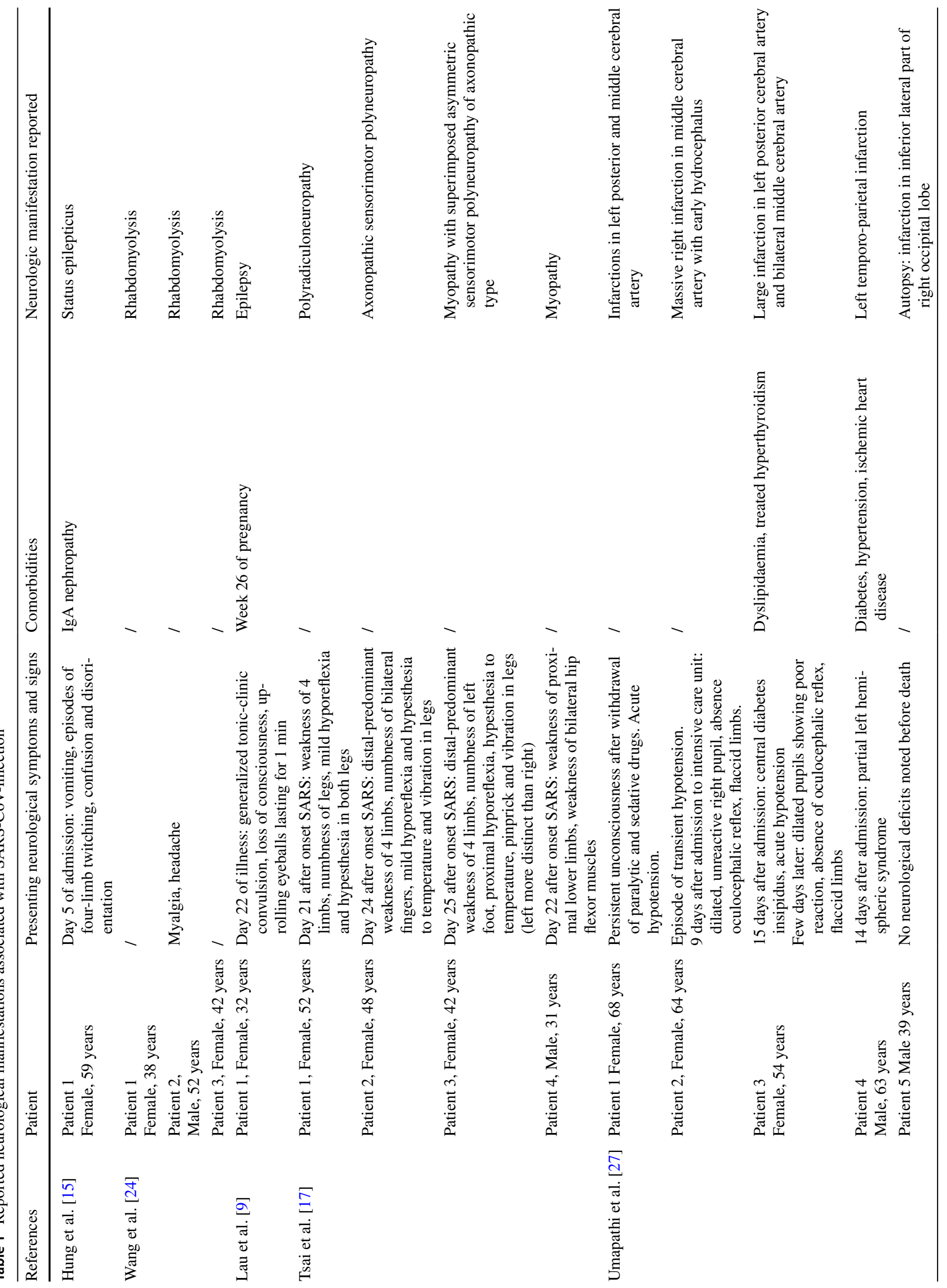




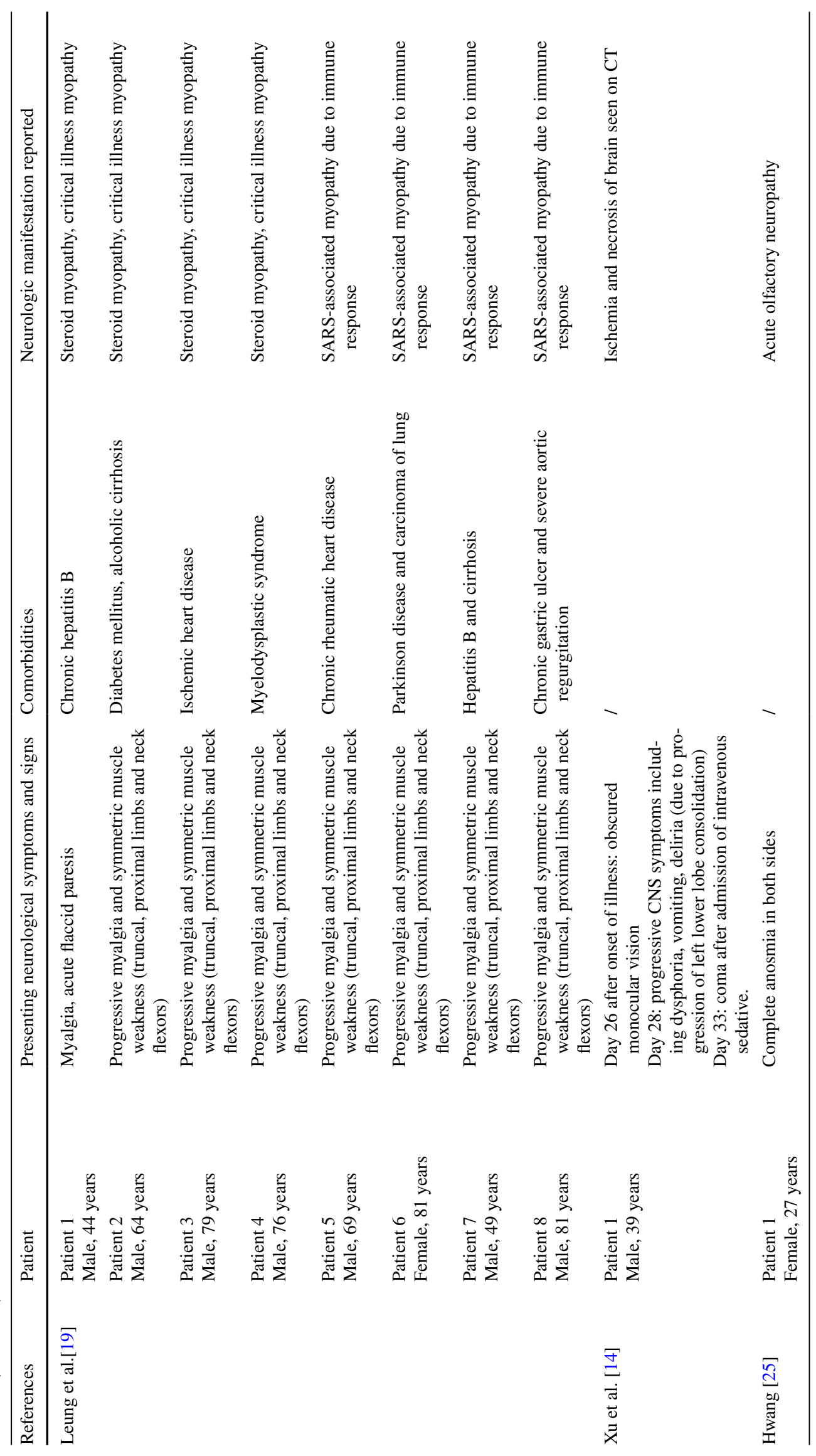




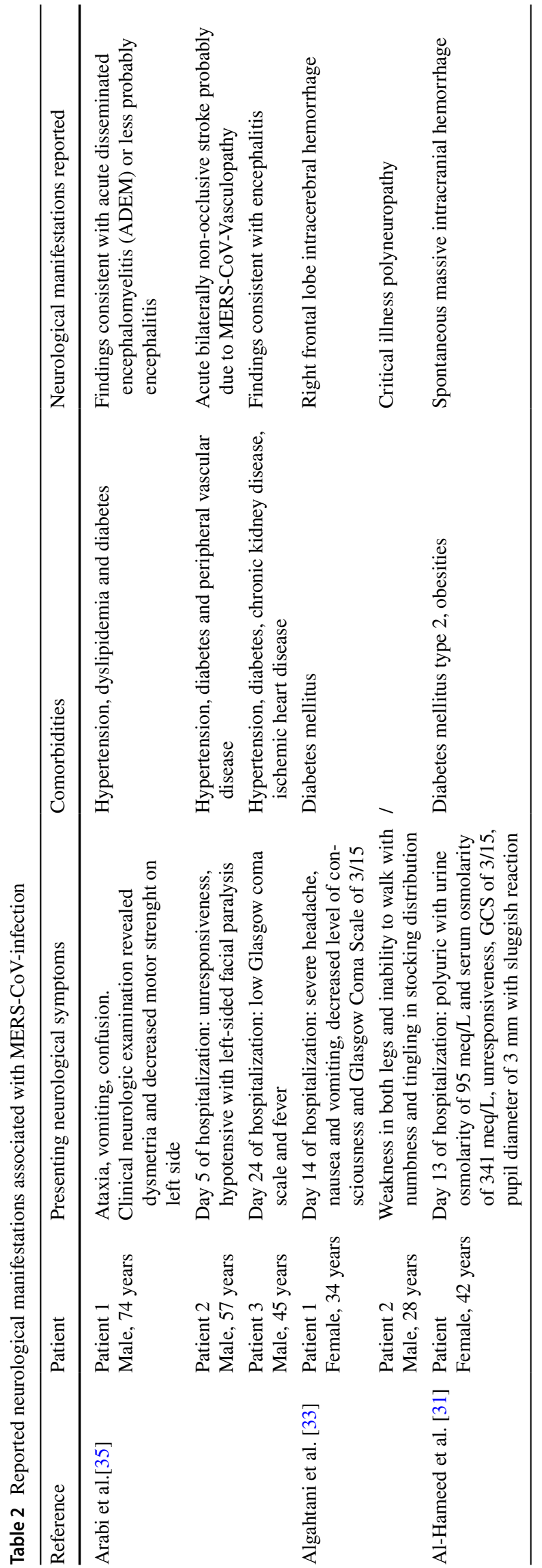

\section{CNS involvement}

Although it is exceptional, the CNS could be involved by MERS-CoV infection, mostly due to auto-immune reaction through autoreactive T-cells which recognize viral and myelin antigens as similar molecules, rather than to viral infection itself [31-33].

A retrospective study in Saudi Arabia stated that about $25 \%$ of MERS patients developed confusion and $8.6 \%$ experienced a seizure [34].

Another case series of three patients with altered level of consciousness varying from confusion to coma, ataxia and focal motor deficit, describes diffuse CNS disease with MRI showing new onset, widespread, bilateral hyperintense lesions on T2-weighted imaging within white matter and subcortical areas of different lobes, basal ganglia, corpus callosum, pons, cerebellum and upper cervical cord with only non-specific increased protein level in CSF.

Final working diagnoses in the three patients were respectively acute disseminated encephalomyelitis, acute bilateral non-occlusive stroke due to MERS-CoV vasculopathy and encephalitis [35].

The negative CSF for MERS-CoV RT-PCR might be due to the timing of the test, the lack of meningeal involvement as shown on MRI, or the virus being located intra-neuronally as reported with SARS-CoV [36].

Intracerebral haemorrhage as result of thrombocytopenia, disseminated intravascular coagulation and platelet dysfunction, is very rare [32].

In 2007, Al-Hameed et al. described the case of a female health care worker who developed a sudden-onset diabetes insipidus and spontaneous massive intracranial haemorrhage with intra-ventricular extension and tonsillar herniation. Her platelet count and coagulation profile did not show any abnormalities. As mentioned in the table, the woman was obese. The severity of MERS in obese patients can be explained as dipeptidyl peptidase 4 (DPP4), a transmembrane protein involved in cell entry of viruses such as MERS-CoV, is overexpressed in obese patients [37, 38].

\section{Neuromuscular disorders}

Only one case of a 28-year-old male, an orthopaedic resident, was reported. After a long stay in intensive care, he suffered from weakness in both legs and inability to walk, along with numbness and tingling in stocking distribution. He was diagnosed with critical illness polyneuropathy [32].

\section{latrogenic neurological pathology during treatment of MERS}

Kim et al. published a case report of four patients who developed neurological manifestations during treatment of 
MERS. A triple antiviral treatment, consisting of subcutaneous pegylated interferon alpha-2a, high-dose oral ribavirin and oral lopinavir/ritonavir, was administered to all patients in the concerned hospital. Characteristics, presentation and diagnosis of the reported patients experiencing neurological symptoms during their treatment of MERS-CoV-infection are summarized in Table 3 [39].

Interferon alpha-2a is a possible causative drug for peripheral neuropathy, sensory neuropathy, vasculitic neuropathy, Bell's palsy, Guillain Barré Syndrome, chronic inflammatory demyelinating polyneuropathy and autonomic polyneuropathy [40-42]. Lopinavir/ritonavir is another possible causative drug for peripheral neuropathy [43]. Ribavirine is not associated with peripheral neuropathy [42].

\section{Neurological manifestations associated with SARS-CoV-2 infection}

Similar to SARS-CoV, SARS-CoV-2 profits from the angiotensin converting enzyme 2 receptor (ACE2-R) to infiltrate in the intracellular space. It has been described that the brain expresses ACE2-R, which have been detected in glial cells and neurons as well as endothelial cells and smooth muscle cells. Therefore, these cells and neurons are potential targets of COVID-19 [44] COVID-19 may reach the central nervous system via the systemic circulation or via the cribriform plate of the ethmoid bone during an early or later phase of the infection as has been reported in SARS-CoV [45] Once the neuronal tissues are reached, the interaction between SARS-CoV-2 and ACE2-R can initiate a cycle of viral growth with co-occurrence of neuronal damage without substantial inflammation as has been seen with cases of SARS-CoV [45].

What does this mean in the clinical setting?

\section{Neurological symptoms in early disease stages}

These are similar to SARS and MERS and merely non-specific including dizziness, nausea, vomiting, and headache $[46,47]$.

Hypogeusia and hyposmia are frequently reported and have become a cardinal factor in the early diagnosis of COVID-19 [46].

Most probably, this is caused by direct invasion of the olfactory bulb when SARS-CoV-2 makes its entry into the skull via the cribriform plate [44].

A recent German report states that it seems to be well distinguishable from a post-infectious olfactory disorder following rhinitis or another upper respiratory tract infection: most of the patients describe a rather sudden, almost complete loss of odour (i.e. mostly anosmia, less often hyposmia). COVID-19 patients are less likely to have other nasal 
symptoms such as nasal breathing obstruction, clinically manifest rhinitis or excessive nasal secretions [48].

\section{Acute Inflammatory Demyelinating Polyneuropathy can precede respiratory disease}

By the end of April 2020, after 4 months of evolving pandemic, Guillain Barré syndrome (GBS) was reported in less than ten COVID-19-patients, but more cases are in press.

Most of the cases occurred in the early stage of COVID19 in patients presenting with only minor respiratory symptoms at onset of GBS. Although it is notable that the disease severity for both the GBS and pneumonia can deteriorate simultaneously [49-51].

One report described GBS in a patient without any respiratory symptoms, but with loss of smell and taste preceding GBS [52].

\section{Cerebrovascular disease during COVID-19}

A variety of cerebrovascular disorders has been recently reported, probably due to endothelial dysfunction, or hypercoagulability in COVID-19 patients.

These disorders include ischemic stroke, intracerebral hemorrhage, and cerebral venous sinus thrombosis [44, 54-56]. Cerebrovascular disease was associated with poor outcome (more respiratory distress, more multi-organ dysfunction, and higher mortality) of COVID-19 patients [56].

\section{Conclusion}

The three major outbreaks of corona viruses (SARS-CoV, MERS-CoV, and SARS-CoV-2) show similarities in neurological manifestations, but also some differences.

Due to the hospital emergency plan and the splitting of hospitals in hot zones versus cold zones to prevent hospital clusters of corona-infection, neurological work up is limited to bedside clinical evaluation, spinal fluid analysis and CT scan. MRI imaging is mostly located in the cold zone, thus limited available for infected patients. A surveillance of neurological manifestations (Guillain-Barré syndrome, meningoencephalitis, stroke...) in these patients is warranted as is surveillance for thromboembolic sequelae (pulmonary embolism, stroke). Many patients present with fever, respiratory symptoms, but also headache, anosmia, dizziness. However, spinal tap is not routinely performed and literature on spinal fluid analysis is very sparse. An explanation for the lack of CSF-data would be that there is currently no validated test to detect SARS-CoV-2 in CSF. A multidisciplinary approach and more neurology consultations in the emergency department and COVID-19-wards could prevent under-diagnosing of neurological complications and thus insufficient treatment. Rapid diagnosis and proper treatment of Guillain-Barré syndrome, critical illness polyneuropathy, stroke, and other neurological disorders could have a positive impact on outcome. As the COVID-19 pandemic is evolving, our knowledge on the neurological manifestations of COVID-19 is also evolving. Although the current pandemic is of a much higher scale than SARS and MERS, and far more neurological cases could be expected, the thusfar available literature about neurological manifestation of COVID-19 is rather limited. This review will hopefully contribute to increased alertness for both neurologists and non-neurologists involved in the care of COVID-19 patients.

\section{Compliance with ethical standards}

Conflict of interest All the authors report no disclosure nor conflict of interest relevant to the manuscript. All authors report no financial disclosure.

Ethical approval This manuscript does not contain any studies with human participants or animals performed by any of the authors.

\section{References}

1. Cascella M, Rajnik M, Cuomo A, Dulebohn SC, Di Napoli R (2020) Features, evaluation and treatment coronavirus (COVID19). StatPearls. Treasure Island (FL): StatPearls Publishing. StatPearls Publishing LLC

2. Cunha CB, Opal SM (2014) Middle East respiratory syndrome (MERS): a new zoonotic viral pneumonia. Virulence 5(6):650-654

3. Lu R, Zhao X, Li J, Niu P, Yang B, Wu H et al (2020) Genomic characterisation and epidemiology of 2019 novel coronavirus: implications for virus origins and receptor binding. The Lancet 395(10224):565-574

4. Bradley BT, Bryan A (2019) Emerging respiratory infections: the infectious disease pathology of SARS, MERS, pandemic influenza, and Legionella. Semin Diagn Pathol 36(3):152-159

5. Hui DSC, Zumla A (2019) Severe acute respiratory syndrome: historical, epidemiologic, and clinical features. Infect Dis Clin North Am 33(4):869-889

6. Desforges M, Le Coupanec A, Dubeau P, Bourgouin A, Lajoie L, Dubé M et al (2019) Human coronaviruses and other respiratory viruses: underestimated opportunistic pathogens of the central nervous system? Viruses. 12(1):14

7. Lau KK, Yu WC, Chu CM, Lau ST, Sheng B, Yuen KY (2004) Possible central nervous system infection by SARS coronavirus. Emerg Infect Dis 10(2):342-344

8. Yeh EA, Collins A, Cohen ME, Duffner PK, Faden H (2004) Detection of coronavirus in the central nervous system of a child with acute disseminated encephalomyelitis. Pediatrics 113(1 Pt 1):e73-e76

9. Arbour N, Day R, Newcombe J, Talbot PJ (2000) Neuroinvasion by human respiratory coronaviruses. J Virol 74(19):8913-8921

10. Desforges M, Le Coupanec A, Stodola JK, Meessen-Pinard M, Talbot PJ (2014) Human coronaviruses: viral and cellular factors involved in neuroinvasiveness and neuropathogenesis. Virus Res 194:145-158 
11. Arabi YM, Balkhy HH, Hayden FG, Bouchama A, Luke T, Baillie JK et al (2017) Middle East Respiratory Syndrome. N Engl J Med 376(6):584-594

12. Singhal T. A Review of Coronavirus Disease-2019 (COVID-19). Indian journal of pediatrics. 2020

13. Gu J, Gong E, Zhang B, Zheng J, Gao Z, Zhong Y et al (2005) Multiple organ infection and the pathogenesis of SARS. J Exp Med 202(3):415-424

14. Xu J, Zhong S, Liu J, Li L, Li Y, Wu X et al (2005) Detection of severe acute respiratory syndrome coronavirus in the brain: potential role of the chemokine mig in pathogenesis. Clin infect Dis 41(8): 1089-1096

15. Hung EC, Chim SS, Chan PK, Tong YK, Ng EK, Chiu RW et al (2003) Detection of SARS coronavirus RNA in the cerebrospinal fluid of a patient with severe acute respiratory syndrome. Clin Chem 49(12):2108-2109

16. Lai SL, Hsu MT, Chen SS (2005) The impact of SARS on epilepsy: the experience of drug withdrawal in epileptic patients. Seizure. 14(8):557-561

17. Tsai LK, Hsieh ST, Chao CC, Chen YC, Lin YH, Chang SC et al (2004) Neuromuscular disorders in severe acute respiratory syndrome. Arch Neurol 61(11):1669-1673

18. Lee N, Hui D, Wu A, Chan P, Cameron P, Joynt GM et al (2003) A major outbreak of severe acute respiratory syndrome in Hong Kong. N Engl J Med 348(20):1986-1994

19. Leung TW, Wong KS, Hui AC, To KF, Lai ST, Ng WF et al (2005) Myopathic changes associated with severe acute respiratory syndrome: a postmortem case series. Arch Neurol 62(7):1113-1117

20. Zochodne DW (2004) SARS, SIRS, and neurological disease. Arch Neurol 61(11):1647-1648

21. de Bock F, Derijard B, Dornand J, Bockaert J, Rondouin G (1998) The neuronal death induced by endotoxic shock but not that induced by excitatory amino acids requires TNF-alpha. Eur J Neurosci 10(10):3107-3114

22. Levy D, Höke A, Zochodne DW (1999) Local expression of inducible nitric oxide synthase in an animal model of neuropathic pain. Neurosci Lett 260(3):207-209

23. Singh U, Scheld WM (1996) Infectious Etiologies of Rhabdomyolysis: three Case Reports and Review. Clin Infect Dis 22(4):642-649

24. Wang J-L, Wang J-T, Yu C-J, Chen Y-C, Hsueh P-R, Hsiao C-H et al (2003) Rhabdomyolysis associated with probable SARS. Am J Med 115(5):421-422

25. Hwang CS (2006) Olfactory neuropathy in severe acute respiratory syndrome: report of A case. Acta Neurol Taiwan 15(1):26-28

26. Dalakas MC, Clark WM (2003) Strokes, thromboembolic events, and IVIg: rare incidents blemish an excellent safety record. Neurology 60(11):1736-1737

27. Umapathi T, Kor AC, Venketasubramanian N, Lim CC, Pang BC, Yeo TT et al (2004) Large artery ischaemic stroke in severe acute respiratory syndrome (SARS). J Neurol 251(10):1227-1231

28. Moldofsky H, Patcai J (2011) Chronic widespread musculoskeletal pain, fatigue, depression and disordered sleep in chronic postSARS syndrome; a case-controlled study. BMC Neurol 11:37

29. Lo YL, Leong HN, Hsu LY, Tan TT, Kurup A, Fook-Chong $S$ et al (2005) Autonomic dysfunction in recovered severe acute respiratory syndrome patients. Canad J Neurol Sci 32(2):264

30. Choi KW, Chau TN, Tsang O, Tso E, Chiu MC, Tong WL et al (2003) Outcomes and prognostic factors in 267 patients with severe acute respiratory syndrome in Hong Kong. Ann Intern Med 139(9):715-723

31. Al-Hameed FM (2017) Spontaneous intracranial hemorrhage in a patient with Middle East respiratory syndrome corona virus. Saudi Med J 38(2):196-200

32. Algahtani H, Subahi A, Shirah B (2016) Neurological complications of middle east respiratory syndrome coronavirus: a report of two cases and review of the literature. Case Rep Neurol Med 2016:3502683

33. Joob B, Wiwanitkit V (2015) Neurologic syndrome due to MERS: is there a possibility that the virus can cross the blood-brain barrier to cause a neurological problem? Ann Trop Med Publ Health $8(5): 231$

34. Saad M, Omrani AS, Baig K, Bahloul A, Elzein F, Matin MA et al (2014) Clinical aspects and outcomes of 70 patients with middle east respiratory syndrome coronavirus infection: a single-center experience in Saudi Arabia. Int J Infect Dis 29:301-306

35. Arabi YM, Harthi A, Hussein J, Bouchama A, Johani S, Hajeer $\mathrm{AH}$ et al (2015) Severe neurologic syndrome associated with Middle East respiratory syndrome corona virus (MERS-CoV). Infection 43(4):495-501

36. Gu J, Korteweg C (2007) Pathology and pathogenesis of severe acute respiratory syndrome. Am J Pathol 170(4):1136-1147

37. Raj VS, Mou H, Smits SL, Dekkers DH, Müller MA, Dijkman R et al (2013) Dipeptidyl peptidase 4 is a functional receptor for the emerging human coronavirus-EMC. Nature 495(7440):251-254

38. Lamers D, Famulla S, Wronkowitz N, Hartwig S, Lehr S, Ouwens DM et al (2011) Dipeptidyl peptidase 4 is a novel adipokine potentially linking obesity to the metabolic syndrome. Diabetes 60(7):1917-1925

39. Kim JE, Heo JH, Kim HO, Song SH, Park SS, Park TH et al (2017) Neurological Complications during Treatment of Middle East Respiratory Syndrome. Journal of clinical neurology (Seoul, Korea). 13(3):227-233

40. Briani C, Chemello L, Zara G, Ermani M, Bernardinello E, Ruggero $S$ et al (2006) Peripheral neurotoxicity of pegylated interferon alpha. A prospective study in patients with HCV. Neurology 67(5):781-785

41. Meriggioli MN, Rowin J (2000) Chronic inflammatory demyelinating polyneuropathy after treatment with interferon-alpha. Muscle Nerve 23(3):433-435

42. Khiani V, Kelly T, Shibli A, Jensen D, Mohanty SR (2008) Acute inflammatory demyelinating polyneuropathy associated with pegylated interferon alpha 2a therapy for chronic hepatitis $\mathrm{C}$ virus infection. World J Gastroenterol 14(2):318-321

43. Ellis RJ, Marquie-Beck J, Delaney P, Alexander T, Clifford DB, McArthur JC et al (2008) Human immunodeficiency virus protease inhibitors and risk for peripheral neuropathy. Ann Neurol 64(5):566-572

44. Baig AM, Khaleeq A, Ali U, Syeda H (2020) Evidence of the COVID-19 virus targeting the CNS: tissue distribution, host-virus interaction, and proposed neurotropic mechanisms. ACS Chem Neurosci 11(7):995-998

45. Netland J, Meyerholz DK, Moore S, Cassell M, Perlman S (2008) Severe acute respiratory syndrome coronavirus infection causes neuronal death in the absence of encephalitis in mice transgenic for human ACE2. J Virol 82(15):7264-7275

46. Mao L, Wang M, Chen S, He Q, Chang J, Hong C, et al. Neurological Manifestations of Hospitalized Patients with COVID19 in Wuhan, China: a retrospective case series study. medRxiv. 2020:2020.02.22.20026500

47. Li YC, Bai WZ, Hashikawa T (2020) The neuroinvasive potential of SARS-CoV2 may play a role in the respiratory failure of COVID-19 patients. J Med Virol 92(6):552-555

48. Lüers JC, Klußmann JP, Guntinas-Lichius O (2020) The Covid-19 pandemic and otolaryngology: What it comes down to? LaryngoRhino- Otologie. 99(5):287-291

49. Zhao H, Shen D, Zhou H, Liu J, Chen S (2020) Guillain-Barré syndrome associated with SARS-CoV-2 infection: causality or coincidence? Lancet Neurol 19(5):383-384

50. El Otmani H, El Moutawakil B, Rafai MA, El Benna N, El Kettani C, Soussi M et al (2020) Covid-19 and Guillain-Barré syndrome: more than a coincidence! Revue neurologique. 176(6):518-519 
51. Ottaviani D, Boso F, Tranquillini E, Gapeni I, Pedrotti G, Cozzio $S$ et al (2020) Early Guillain-Barré syndrome in coronavirus disease 2019 (COVID-19): a case report from an Italian COVIDhospital. Neurol Sci 2020:1-4

52. Scheidl E, Canseco DD, Hadji-Naumov A, Bereznai B (2020) Guillain-Barre syndrome during SARS-CoV-2 pandemic: a case report and review of recent literature. Journal of the peripheral nervous system: JPNS. https://doi.org/10.1111/jns.12382

53. Goldberg MF, Goldberg MF, Cerejo R, Tayal AH (2020) Cerebrovascular Disease in COVID-19. AJNR American journal of neuroradiology. https://doi.org/10.3174/ajnr.A6588

54. Hess DC, Eldahshan W, Rutkowski E (2020) COVID-19-related stroke. Translational stroke research. 11(3):322-325
55. Hughes C, Nichols T, Pike M, Subbe C, Elghenzai S (2020) Cerebral venous sinus thrombosis as a presentation of COVID-19. European J Case Rep int Med 7(5):001691

56. Pranata R, Huang I, Lim MA, Wahjoepramono PEJ, July J (2020) Impact of cerebrovascular and cardiovascular diseases on mortality and severity of COVID-19 - systematic review, meta-analysis, and meta-regression. J Stroke Cerebrovasc Dis 2020:104949

Publisher's Note Springer Nature remains neutral with regard to jurisdictional claims in published maps and institutional affiliations. 